\title{
A nomogram for predicting in-hospital mortality in acute type A aortic dissection patients
}

\author{
Guifang Yang ${ }^{1,2}$, Yang Zhou ${ }^{1,2}$, Huaping $\mathrm{He}^{1,2}$, Xiaogao Pan ${ }^{1,2}$, Xizhao $\mathrm{Li}^{3}$, Xiangping Chai ${ }^{1,2}$ \\ ${ }^{1}$ Department of Emergency Medicine, the Second Xiangya Hospital, Central South University, Changsha 410011, China; ${ }^{2}$ Emergency Medicine \\ and Difficult Diseases Institute, Central South University, Changsha 410011, China; ${ }^{3}$ Department of Cardiovascular Surgery, the Second Xiangya \\ Hospital, Central South University, Changsha 410011, China \\ Contributions: (I) Conception and design: G Yang; (II) Administrative support: X Chai; (III) Provision of study materials or patients: G Yang; (IV) \\ Collection and assembly of data: Y Zhou, H He, X Pan, X Li; (V) Data analysis and interpretation: X Chai; (VI) Manuscript writing: All authors; (VII) \\ Final approval of manuscript: All authors. \\ Correspondence to: Xiangping Chai. Department of Emergency Medicine, the Second Xiangya Hospital, Central South University, 139 Renmin Road, \\ Changsha 410011, China. Email: chaixiangping@csu.edu.cn.
}

\begin{abstract}
Background: Although there are several biomarkers for identifying in-hospital mortality in acute type A aortic dissection (AAD), timely as well as perfect prediction in-hospital mortality is still not attained. Herein, we intend to develop as well to validate an in-hospital mortality risk independent predictive nomogram for AAD patients.

Methods: From January 2014 to December 2018, 703 individuals with AAD were involved in this study. They were indiscriminately categorized into training $(n=520)$ and validation $(n=183)$ sets. The univariate and multivariate analyses were used to screen in-hospital mortality predictors from the entire training set data. The predictors were used to establish a nomogram which was confirmed via internal as well as external authentication. This validation included discriminative capacity defined by the receiver operating characteristic (ROC) curve area under the curve (AUC) and the predictive precision via calibration curves.

Results: There was $33.43 \%$ in-hospital mortality overall incidence. The uric acid, D-dimer, C-reactive protein and management were individually related to in-hospital mortality as per multivariate logistic regression. On the basis of four variables with internal of AUC 0.901 and external validation of AUC 0.903, a nomogram was established. Calibration plots showed that the predicted and actual in-hospital mortality probabilities were fitted well on both internal and external validation.
\end{abstract}

Conclusions: This recommended nomogram can calculate the specific possibility of in-hospital mortality with good precision, high discrimination, and probable clinical application in AAD patients.

Keywords: Acute aortic dissection; nomogram; in-hospital mortality

Submitted Oct 24, 2019. Accepted for publication Dec 30, 2019.

doi: $10.21037 /$ jtd.2020.01.41

View this article at: http://dx.doi.org/10.21037/jtd.2020.01.41

\section{Introduction}

Acute type A aortic dissection (AAD), a fatal aortic sickness has a high death rate and morbidity which demands prompt examination and treatment $(1,2)$. From $1 \%$ to $2 \%$ of patients with AAD die per hour for the first 24 48 hours (3). Unfortunately, there is still a lack of effective methods to accurately predict the short-term results of these patients. Recently, there has been a countless pursuit to ascertain the severity outcome of $\mathrm{AAD}$, but, their efficacy in predicting in-hospital mortality remains controversial $(4,5)$. Presently, in spite of extensive appreciation of the significance of in-hospital mortality, there is a lack of a perfect predictive method to identify in-hospital mortality in time to induce prompt intervention. A nomogram can offer, convenient form, precise risk estimate of patient individual clinical results grounded on major factors $(6,7)$. Currently, to identify several disease diagnosis and 
management strategies, nomograms have been suggested $(8,9)$. The aim of this research is to define in-hospital mortality risk potential predictors and to build its predictive nomogram prototype, that will be important in detecting high-risk patients and subsequently guide them through psychotherapy and choosing the proper therapy, upon AAD.

\section{Methods}

\section{Study design and settings}

This is a retrospective observational study design. Medical records of AAD patients admitted to the Second Xiangya Hospital of Central South University from January 2014 to December 2018 were investigated. It was appraised and ratified by the hospital ethics committee, and as a retrospective study, the informed consent was waived.

Stanford standards were used to categorize AAD form and the diagnosis was established via computed tomography angiography (CTA) or magnetic resonance angiography (MRA) (10). AAD patients with a time interval of $\leq 14$ days from the onset of symptoms to hospital admission were included in the present study. Exclusion criteria included: (I) diagnosis with pregnancy; (II) presence of intramural hematoma; (II) presence of symptoms for more than 14 days.

\section{Collection of data}

Each patient's clinical and laboratory information was acquired through electronic medical registers. Elementary clinical information comprised date of birth, sex, body mass index (BMI), the presence of hypertension, diabetes, stroke, atherosclerosis, Marfan syndrome, chronic renal insufficiency (CRI), smoking, symptom, blood pressure, pulse pressure, and management.

Laboratory variables included white blood cell (WBC), neutrophil ratio (N\%), platelet (PLT), hemoglobin $(\mathrm{Hb})$, alanine transaminase (ALT), aspartate aminotransferase (AST), albumin (ALB), total bilirubin (TB), direct bilirubin $(\mathrm{DB})$, creatinine $(\mathrm{Cr})$, blood urea nitrogen (BUN), uric acid (UA), estimated glomerular filtration rate (eGFR), creatine kinase $(\mathrm{CK})$, creatine kinase-MB $(\mathrm{CK}-\mathrm{MB})$, troponin $\mathrm{T}$ (TNT), B-type natriuretic peptide (BNP), D-dimer (DDR), and $\mathrm{C}$-reactive protein (CRP). Venous blood was drawn from all patients after admission. Plasma was obtained after rapid centrifugation and was immediately stored at $-20{ }^{\circ} \mathrm{C}$ for further analysis. Serum biomarkers were determined using commercial kits and an automated biochemical analyzer. Once admitted all the above were analyzed the main laboratory of the Second Xiangya Hospital of Central South University.

\section{Clinical endpoint}

The clinical study was terminated upon death in the hospital.

\section{Statistical analysis}

Statistical results were presented as the mean \pm standard deviation for normal data whereas non-normal data, interquartile range (IQR) and median were used. The categorical variables were presented as percentages and numbers. The non-normally distributed continuous variables Wilcoxon Mann-Whitney tests in addition to unpaired Student $t$-tests for normally distributed continuous variables were utilized to establish the correlations among the survivor and the non-survivor groups. Fisher's exact/ Chi-squared tests were utilized to evaluate clustered variables.

The initial dataset was indiscriminately divided into training and validating set at a 3:1 ratio and was utilized in producing and authenticating the model, respectively. To identify the in-hospital death risk features among $\mathrm{AAD}$ victims, the study was carried out using multivariate regression in the training set. In-hospital mortality was used as reaction variable adopting a binomial distribution, and covariates with lesser than $0.05 \mathrm{P}$ values were incorporated in the model.

An in-hospital mortality AAD patients' predictive nomogram was established from clinical significant variables. The validation involved two events: internal and external authentication. To begin with, internal validation was completed through a bootstrap process using 500 resamples. The discrimination capacity was studied via the receiver operating characteristic (ROC) curve area under the curve (AUC). In addition, a calibration curve was drawn to quantify the consistency of nomogram predicted chances and the experienced in-hospital mortality frequency. Secondly, external authentication was executed in the validation group and the nomogram employed for individual patient validation. To evaluate the nomogram prototype discriminative achievements and predictive precision, AUC and calibration plot was made. It was found out that the $\mathrm{P}$ values were all 2 -sided and $\mathrm{P}<0.05$ 


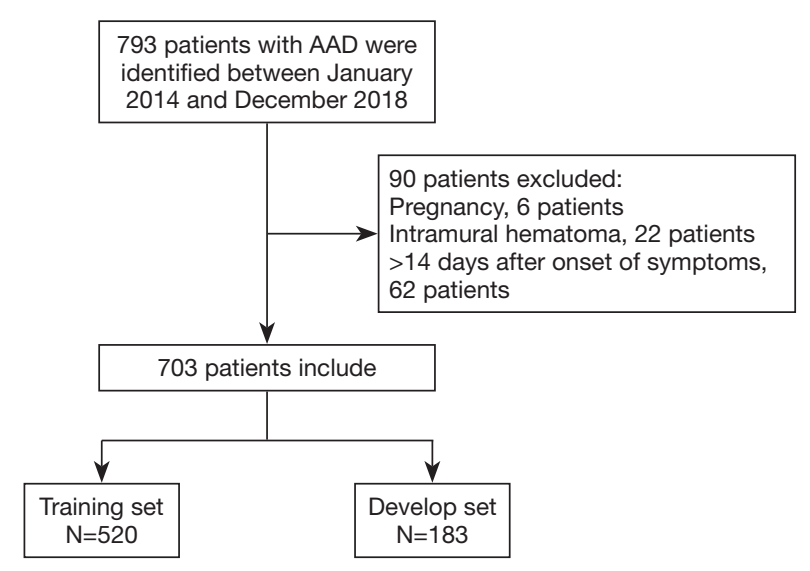

Figure 1 Flow chart of patient enrollment.

was statistically significant. R (http://www.R-project.org) and Empowerstates (http://www.empowerstates.com, X\&Y Solution, Inc, Boston, MA, USA) software were used to carry out all the statistical analyses.

\section{Results}

\section{Study population and clinical characteristics of the study cobort}

Seven hundred and ninety-three patients were identified with a diagnosis of AAD. Among them, 6 patients were expectant, 22 patients were diagnosed intramural hematoma, and 62 patients got admitted after 24 hours of symptoms origin of were excluded from the analysis. Here, a cohort of 703 AAD victims was incorporated, of which three-quarters were indiscriminately allocated to the training set $(\mathrm{n}=520)$ to generate a predictive nomogram model, while the 183 left were apportioned to the validation set to assess the model success (Figure 1). There was no significant variation in most elementary clinical information and laboratory variables among the training and validation groups (Table 1). The AAD in-hospital mortality between the two groups was similar, $175(33.65 \%)$ in the training set and $60(32.79 \%)$ in the validation set, respectively.

\section{Independent predictors of in-hospital mortality for $A A D$ victims}

Univariate analysis indicated that age, SBP, DBP, pulse pressure, WBC, N\%, PLT, ALT, AST, ALB, Cr, BUN, UA, eGFR, CK, CK-MB, TNT, BNP, DDR, CRP, atherosclerosis, CRI, and management were associated with in-hospital mortality for the training set as shown in Table 2. The above were incorporated in multivariate regression analysis to monitor the important in-hospital mortality predictors. This analysis outcome disclosed four selfsufficient predictor variables including UA, DDR, CRP, and management for in-hospital mortality AAD victims (Table 3).

\section{Development of in-hospital mortality predictive nomogram for AAD patients}

The predictive nomogram (Figure 2), was developed from the four independent in-hospital mortality predictors. Individually, subsets in these variables were allocated a count on the point set of scales. Once we added up the cumulative score and tracing on the overall point scale, we plotted a straight line down to obtain in-hospital mortality predicted probability. A higher score of overall points revealed a better in-hospital mortality chance.

\section{Internal validation of the predictive nomogram}

Upon exposure to internal authentication, the predictive nomogram validated a perfect discriminative capacity of AUC 0.901 (95\% CI: 0.869-0.933) (Figure 3A). The standard curve disclosed that predicted nomogram possibilities of in-hospital sensibly estimated actual probabilities (Figure 3B).

\section{External validation of the predictive nomogram}

External authentication was achieved by comparing the predictive nomogram and individual actual possibility in the authentication group. For validation group, predictive nomogram AUC as 0.903 (95\% CI: 0.858-0.967) (Figure $4 A$ ). The standardization plot revealed that there was perfect synchronization among predicted and real possibilities (Figure 4B).

\section{Clinical application of the nomogram}

Meanwhile, we chose two AAD victims as specimens for the nomogram presentation. The levels of UA, DDR, and CRP in the first patient were $926.1 \mu \mathrm{mol} / \mathrm{L}$ (84 points), $3.3 \mu \mathrm{g} / \mathrm{mL}$ ( 2 points), $122 \mathrm{mg} / \mathrm{L}$ (18 points), respectively. His medical treatment at 100 points. There were 204 estimated points nomogram count and above 0.9 probability of in-hospital mortality. The patient had a high chance of achieving in-hospital mortality. In fact, he acquired 
Table 1 Baseline characteristics of the patients in training set and validation set

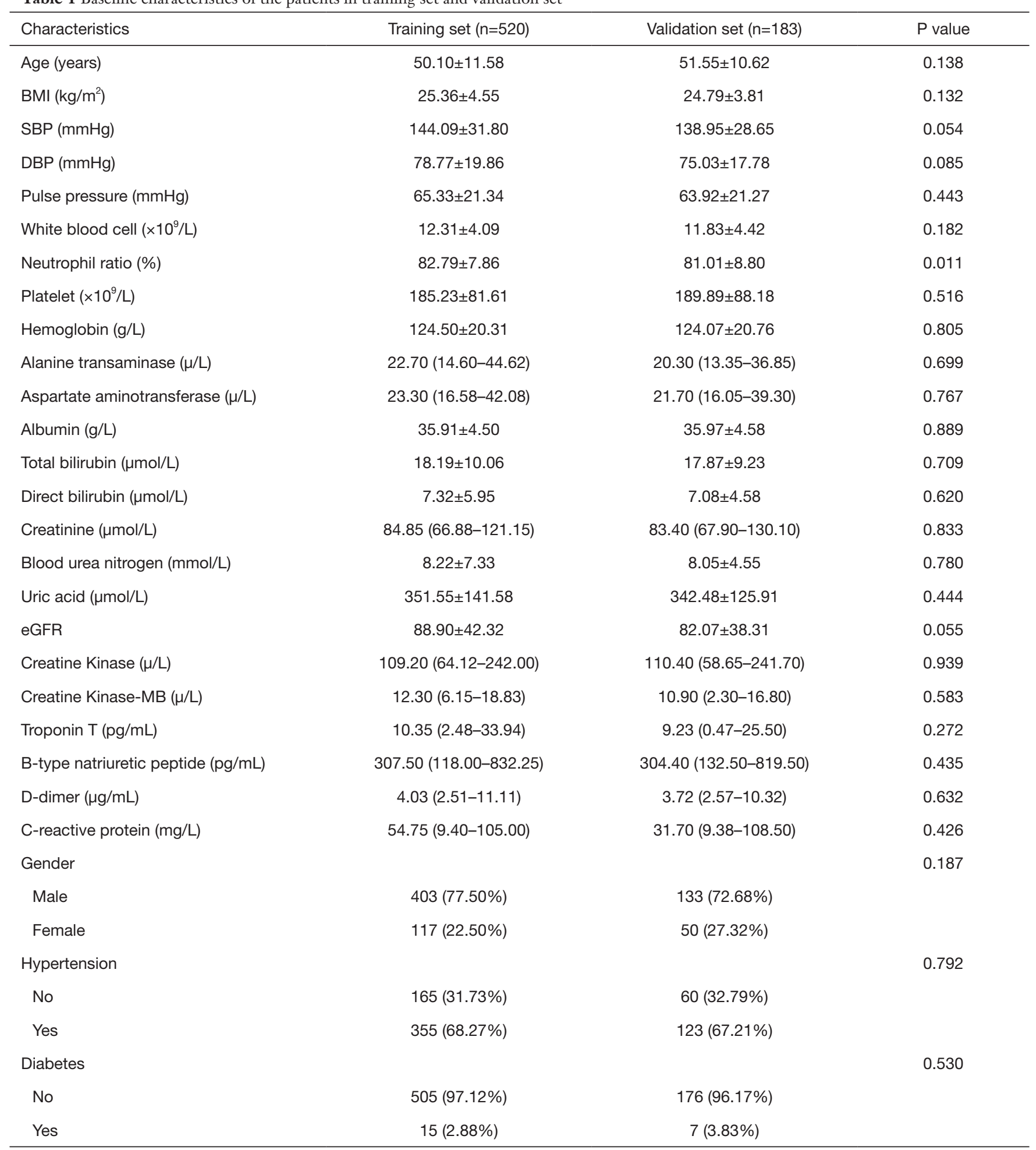

Table 1 (Continued) 
Table 1 (Continued)

\begin{tabular}{|c|c|c|c|}
\hline Characteristics & Training set $(n=520)$ & Validation set $(n=183)$ & $P$ value \\
\hline No & 507 (97.50\%) & 172 (93.99\%) & \\
\hline Yes & $13(2.50 \%)$ & $11(6.01 \%)$ & \\
\hline Atherosclerosis & & & 0.395 \\
\hline Yes & $38(7.31 \%)$ & $10(5.46 \%)$ & \\
\hline Marfan syndrome & & & 0.354 \\
\hline No & 507 (97.50\%) & $176(96.17 \%)$ & \\
\hline Yes & $13(2.50 \%)$ & $7(3.83 \%)$ & \\
\hline Yes & $13(2.50 \%)$ & $5(2.73 \%)$ & \\
\hline Smoking & & & 0.806 \\
\hline No & $373(71.73 \%)$ & $133(72.68 \%)$ & \\
\hline Yes & $147(28.27 \%)$ & $50(27.32 \%)$ & \\
\hline Symptom & & & 0.021 \\
\hline Chest pain & $433(83.27 \%)$ & $148(80.87 \%)$ & \\
\hline Back pain & $18(3.46 \%)$ & $4(2.19 \%)$ & \\
\hline Abdominal pain & $23(4.42 \%)$ & $2(1.09 \%)$ & \\
\hline Mortality & & & 0.831 \\
\hline Survivor & $345(66.35 \%)$ & $123(67.21 \%)$ & \\
\hline Non-survivor & $175(33.65 \%)$ & $60(32.79 \%)$ & \\
\hline
\end{tabular}

in-hospital mortality in admission. The $2^{\text {nd }}$ patient with surgical treatment (0 points). The levels of UA, DDR and CRP were $89.7 \mu \mathrm{mol} / \mathrm{L}$ (8 points), $9.24 \mu \mathrm{g} / \mathrm{mL}$ (6 points), $68.1 \mathrm{mg} / \mathrm{L}$ (27 points), respectively. The nomogram was 41 points, and there was $<0.1$ in-hospital mortality probability. This patient had low chances of achieving in-hospital mortality. Besides, he did not acquire in-hospital mortality.

\section{Discussion}

Timely diagnosis of in-hospital mortality high-risk patients can help to manage a disease, improve patient-clinician interaction, and facilitate the patient's prognosis. However, up to now, the timely or perfect diagnostic prototype for in-hospital mortality in AAD patients is lacking. The 
Table 2 Univariate analyses of factors associated with in-hospital mortality in the training set

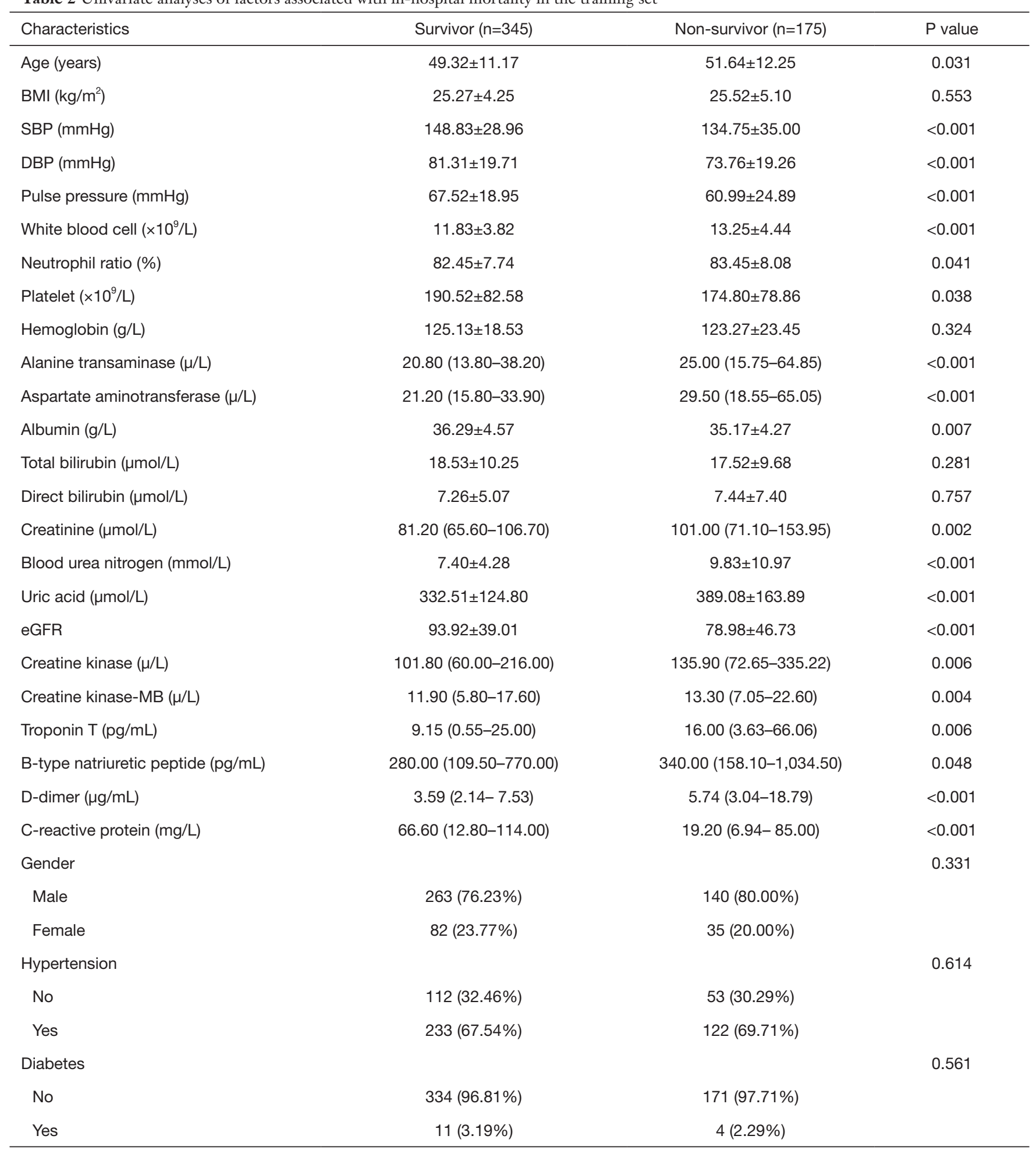

Table 2 (Continued) 
Table 2 (Continued)

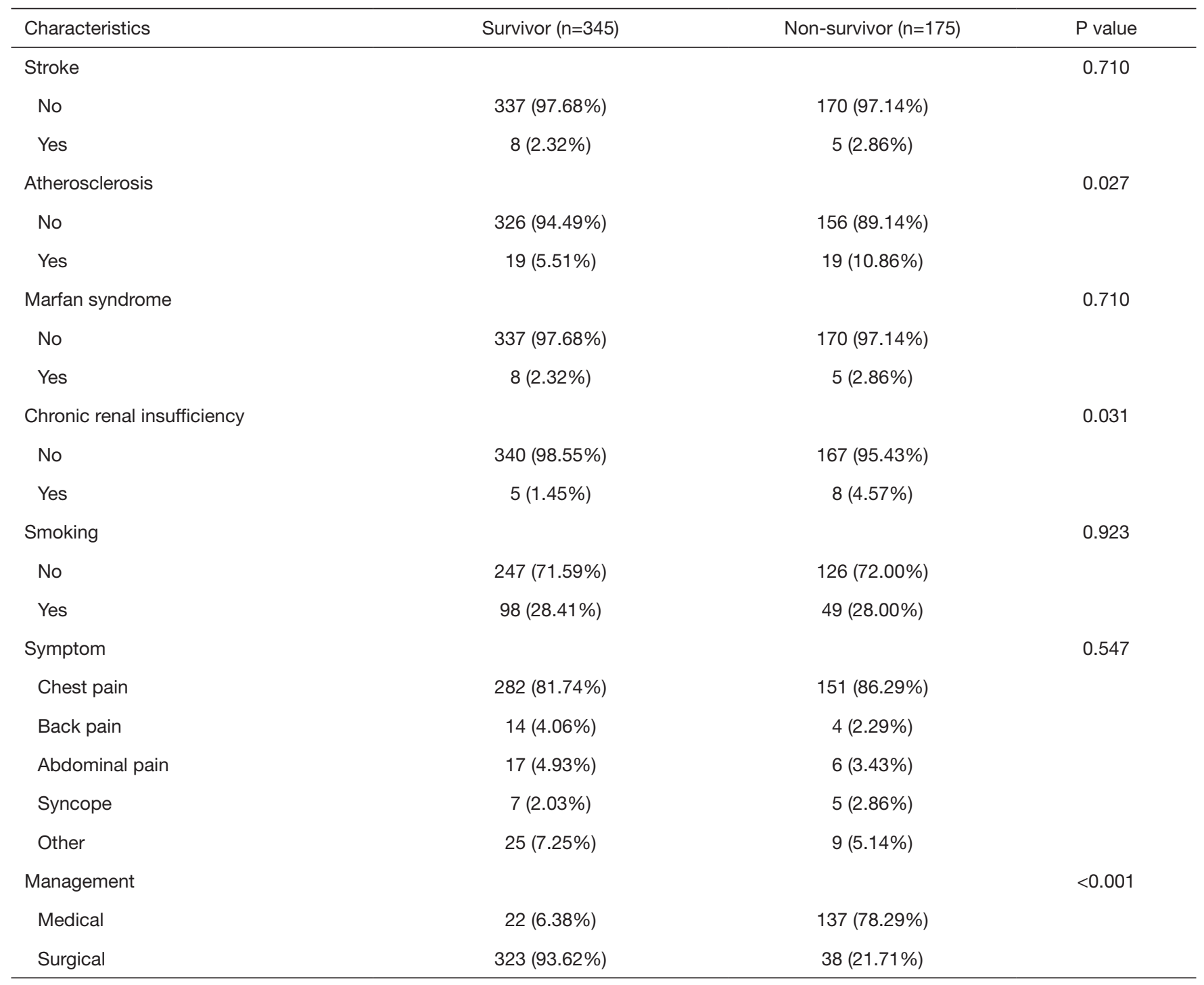

importance of consistent available clinical variables for timely identification of in-hospital mortality has attracted scientific attention. Here, we targeted to develop as well as to authenticate a predictive nomogram for the in-hospital mortality risk in $\mathrm{AAD}$ patients.

To construct the nomogram, the in-hospital mortality independent predictive aspects in $\mathrm{AAD}$ patients were first identified. Through the multivariate logistic regression analysis, the in-hospital mortality independent predictors shown to be UA, DDR, CRP, and management. Similar to earlier studies $(11,12)$, it was shown that medical treatment could contribute to the development of in-hospital mortality.

Evidently, UA, an end product of purine metabolism that is a resilient independent predictor for long-standing mortality in cardiac-related disorders $(13,14)$. Previous studies had revealed that elevated levels of UA were correlated to a higher risk of in-hospital mortality for cardiovascular disease victims. Jiang et al. (15) found that serum uric acid is associated with aortic dissection in Chinese men. A meta-analysis provides evidence that SUA levels are significantly higher among patients with aortic dissection than those in controls. Elevated SUA levels may contribute to the pathogenesis of aortic dissection (16). Experimental and clinical studies have evidenced several mechanisms through which elevated UA level exerts deleterious effects on cardiovascular health 
Table 3 Multivariable logistic regression for in-hospital mortality

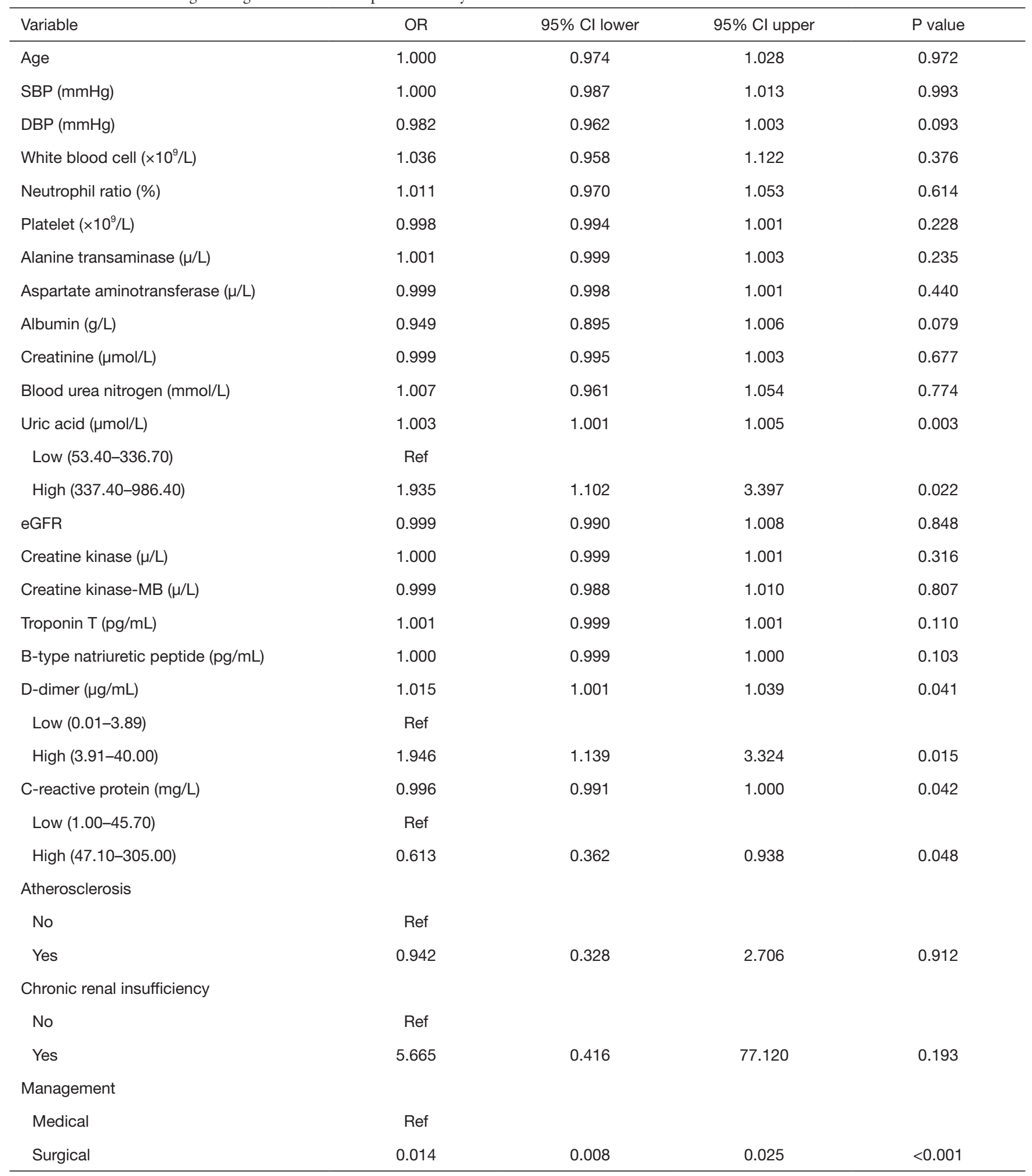




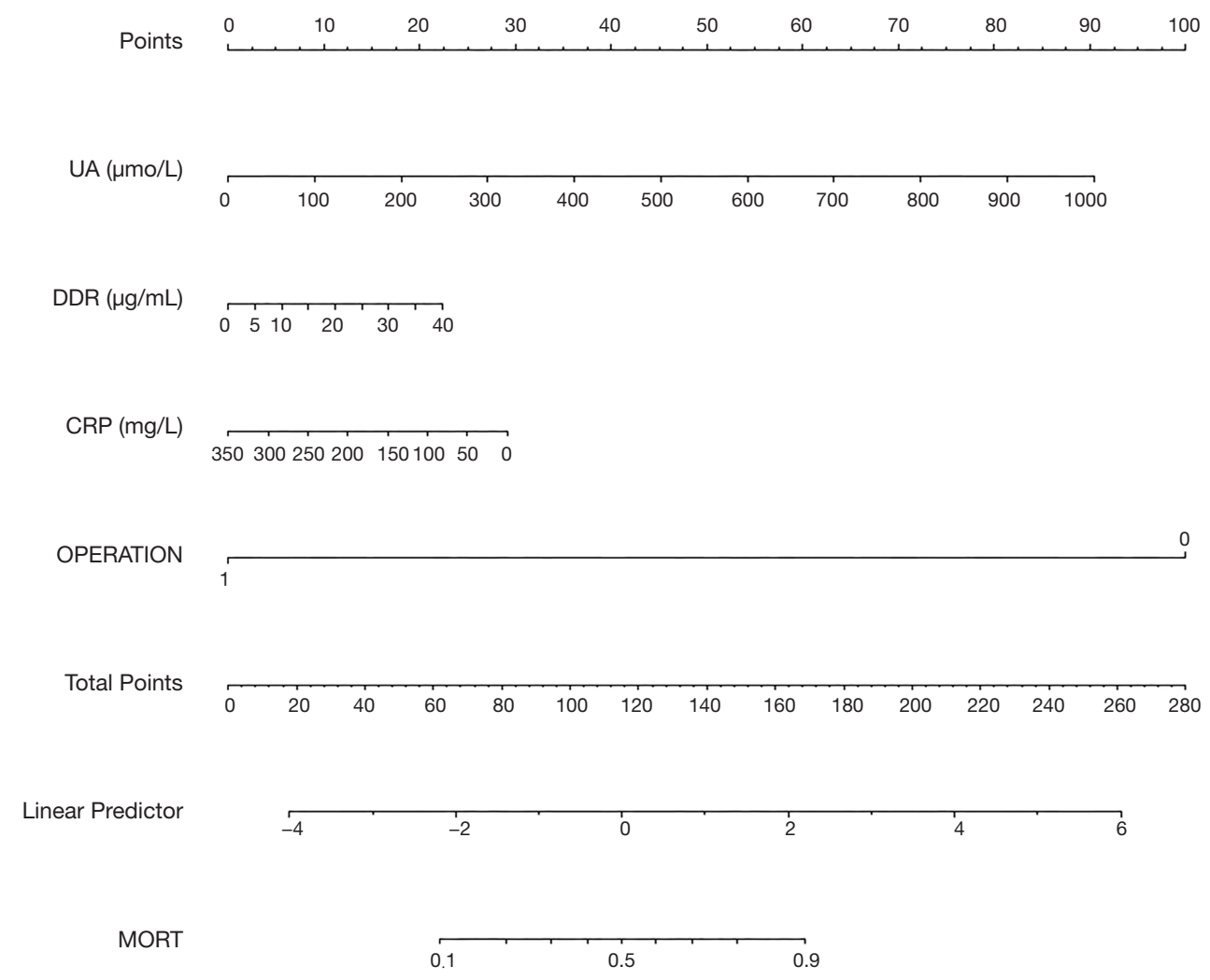

Figure 2 Nomogram model for predicting the risk of in-hospital in patients with AAD. AAD, acute type A aortic dissection; UA, uric acid; DDR, D-dimer; CRP, C-reactive protein; OPERATION, 0= medical, 1= surgical.
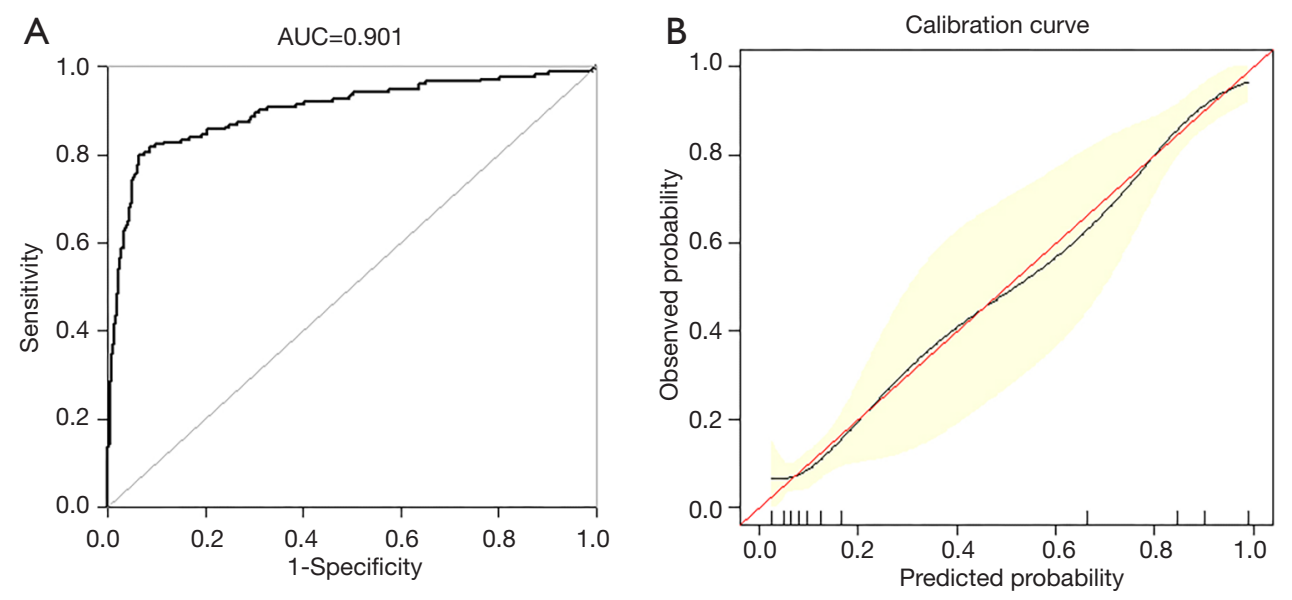

Figure 3 Internal validation of nomogram in the training set. (A) Discrimination: AUC of the ROC curve was 0.901 (95\% CI, 0.8690.933). (B) Nomogram calibration curve. The red line indicates perfect prediction by an ideal model. The black line depicts the model's performance. ROC, receiver operating characteristic; AUC, area under the curve. 

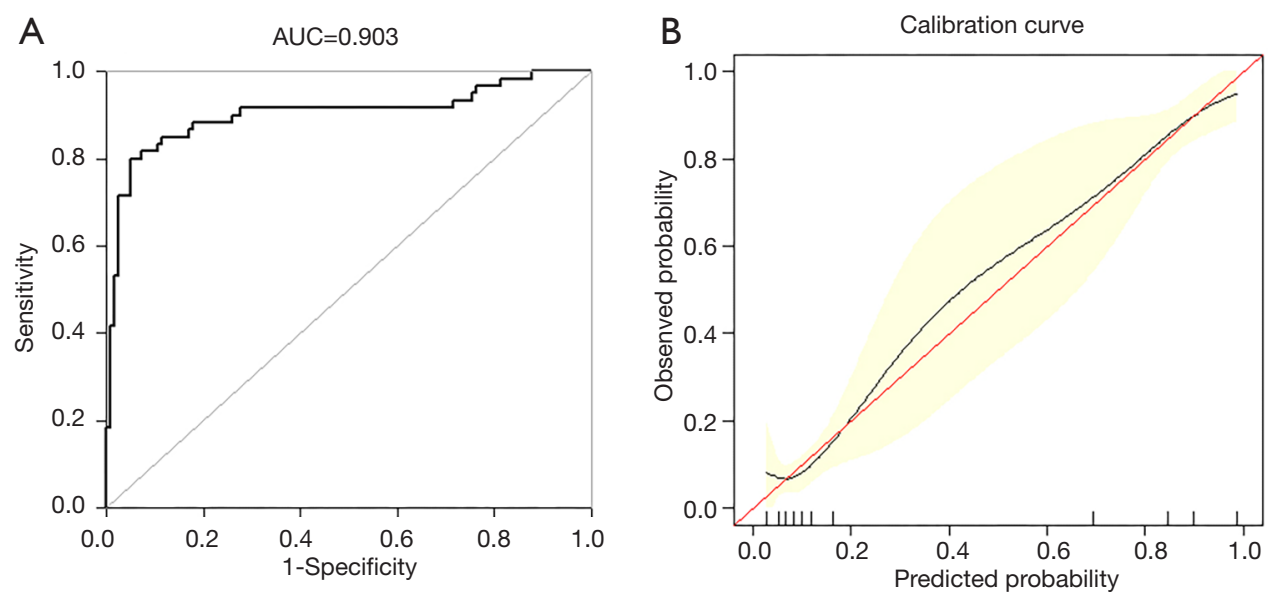

Figure 4 External validation of nomogram in the validation set. (A) Discrimination: AUC of the ROC curve was 0.903 (95\% CI: $0.858-$ 0.967). (B) Nomogram calibration curve. The red line indicates perfect prediction by an ideal model. The black line depicts the model's performance. ROC, receiver operating characteristic; AUC, area under the curve.

including increased oxidative stress, reduced availability of nitric oxide and endothelial dysfunction, promotion of local and systemic inflammation. Hong et al. (17) revealed hyperuricemia induces endothelial dysfunction via mitochondrial $\mathrm{Na}+/ \mathrm{Ca} 2+$ exchanger-mediated mitochondrial calcium overload. Esen et al. (18) revealed that uric acid as a marker of oxidative stress in dilatation of the ascending aorta. In the present research, for AAD patients in-hospital mortality, UA was of predictive significance.

In AAD patients, D-dimer, a cross-linked fibrin degradation yield, is raised (19). Recently, a number of studies estimated the $\mathrm{D}$-dimer levels prognostic value of admitted AAD patients and stated that high levels of D-dimer were related to reduced short-range outcomes $(19,20)$. Our previous study also found that elevated admitted based D-dimer levels can predict a higher risk of in-hospital mortality (21). Besides, when levels of serum D-dimer are high may be a manifestation of more severe and extensive tearing. As in earlier studies, this study maintained that there is a high predictive value of DDR for in-hospital mortality risk.

CRP, a well-known profound systemic inflammation marker, is able to stimulate the clotting system resulting in weakened oxidant defense hence endothelial dysfunction $(22,23)$. AAD is related to inflammatory response, thrombosis, and oxidative pressure demonstrated via a major rise in inflammatory indicators, like CRP (24). AlMahameed et al. (25) suggested that patients with aortic dissection have the highest CRP levels at presentation. Elevated CRP independently predicted higher lasting mortality for AAD patients. Nevertheless, Sakakura et al. (26) found that primary levels of CRP weren't linked to adverse activities, peak CPR intensities were considerably associated with adverse activities. The highest levels of CRP were a superior indicator of primary levels of CRP in AAD type B victims risk stratification. Since it takes a few days to attain CRP peak, primary levels of CRP may not reveal entire aortic dissection severity. Okina et al. (27) also conclude that cardiovascular events cannot be predicted by a CRP value obtained at a single time point, recognition of the overall CRP pattern is far more important. It is interesting that in our research, CRP admission low levels were significantly predictive for AAD in-hospital mortality. These varying outcomes may be due to diverse participants or CRP levels were measured at admission rather than the peak value of monitoring.

According to us, this is the first predictive nomogram for in-hospital mortality risk in AAD patients. It is grounded in four achievable and cost-effective variables that allow ease in determining personalized in-hospital mortality risk evaluations for $\mathrm{AAD}$ patients. The training set nomogram prediction AUC was 0.901 and that for validation set was 0.903 . It was revealed by the standardization plots that the predictive nomogram fitted perfectly to the actual inhospital mortality likelihood on the internal as well as external authentication. These outcomes propose that the predictive nomogram possessed necessary discriminative 
strength and worthy accuracy to predict in-hospital mortality in AAD patients.

Moreover, this study has a few limitations. Firstly, there was only one center in this study. However, even though the training and validation set came from the same institution, this is a tolerable process of developing and authenticating nomogram once the external cohort is non-existing. Nevertheless, the nomogram requires validation from other centers to check its uniqueness. Secondly, there were zero follow-up parameters for nomogram assimilated variables. Yet, the study's goal was to project in-hospital mortality risk from the admission clinical features to bring opportune intervention. Lastly, there was significant variation in a few variables among the training and validation groups. However, these patients were randomly distributed by $\mathrm{R}$ software between the two groups, which ensure that there are no significant differences in most variables like the outcome.

\section{Conclusions}

In summary, this proposed nomogram can determine specifically in-hospital mortality risk with clear accuracy, perfect discrimination, and probable clinical application in $\mathrm{AAD}$ patients.

\section{Acknowledgments}

Funding: This manuscript was supported by the National Natural Science Foundation of China (81471896), Key Research and Development Program of Hunan Province (2019SK2022), Hunan Health and Family Planning Commission Project (No. 20200063).

\section{Footnote}

Conflicts of Interest: The authors have no conflicts of interest to declare.

Ethical Statement: The authors are accountable for all aspects of the work in ensuring that questions related to the accuracy or integrity of any part of the work are appropriately investigated and resolved. The Ethics Committee of the Second Xiangya Hospital, Central South University (Changsha, China) approved this study and because of the study retrospective nature (No. 2018S052), informed consent was omitted.
Open Access Statement: This is an Open Access article distributed in accordance with the Creative Commons Attribution-NonCommercial-NoDerivs 4.0 International License (CC BY-NC-ND 4.0), which permits the noncommercial replication and distribution of the article with the strict proviso that no changes or edits are made and the original work is properly cited (including links to both the formal publication through the relevant DOI and the license). See: https://creativecommons.org/licenses/by-nc-nd/4.0/.

\section{References}

1. Yang GF, Sheng LJ, Peng W, et al. Comorbidity: A novel insight to aortic dissection. Int J Cardiol 2016;207:53-54.

2. Erbel R, Aboyans V, Boileau C, et al. 2014 ESC Guidelines on the diagnosis and treatment of aortic diseases:

Document covering acute and chronic aortic diseases of the thoracic and abdominal aorta of the adult. The Task Force for the Diagnosis and Treatment of Aortic Diseases of the European Society of Cardiology (ESC). Eur Heart J 2014;35:2873-926.

3. Nienaber CA, Clough RE. Management of acute aortic dissection. Lancet 2015;385:800-11.

4. Lederle FA, Powell JT, Nienaber CA. Does intensive medical treatment improve outcomes in aortic dissection? BMJ 2014;349:g5288.

5. Chiappini B, Schepens M, Tan E, et al. Early and late outcomes of acute type A aortic dissection: analysis of risk factors in 487 consecutive patients. Eur Heart J 2005;26:180-6.

6. Lamberink HJ, Otte WM, Geerts AT, et al. Individualised prediction model of seizure recurrence and long-term outcomes after withdrawal of antiepileptic drugs in seizurefree patients: a systematic review and individual participant data meta-analysis. Lancet Neurol 2017;16:523-31.

7. Fan Z, Li Y, Ji H, et al. Nomogram Model to Predict Cardiorenal Syndrome Type 1 in Patients with Acute Heart Failure. Kidney Blood Press Res 2018;43:1832-41.

8. Tendulkar RD, Agrawal S, Gao T, et al. Contemporary Update of a Multi-Institutional Predictive Nomogram for Salvage Radiotherapy After Radical Prostatectomy. J Clin Oncol 2016;34:3648-54.

9. Wu J, Qiu J, Jiang W, et al. Development and validation of a nomogram predicting the probability of type a aortic dissection at a diameter below $55 \mathrm{~mm}$ : A retrospective cohort study. Int J Surg 2018;60:266-72.

10. Bossone E, LaBounty TM, Eagle KA. Acute aortic 
syndromes: diagnosis and management, an update. Eur Heart J 2018;39:739-749d.

11. Mussa FF, Horton JD, Moridzadeh R, et al. Acute Aortic Dissection and Intramural Hematoma: A Systematic Review. JAMA 2016;316:754-63.

12. Mehta RH, Suzuki T, Hagan PG, et al. Predicting death in patients with acute type a aortic dissection. Circulation 2002;105:200-6.

13. Rahimi-Sakak F, Maroofi M, Rahmani J, et al. Serum uric acid and risk of cardiovascular mortality: a systematic review and dose-response meta-analysis of cohort studies of over a million participants. BMC Cardiovasc Disord 2019;19:218.

14. Feig DI, Kang DH, Johnson RJ. Uric acid and cardiovascular risk. N Engl J Med 2008;359:1811-21.

15. Jiang WL, Qi X, Li X, et al. Serum uric acid is associated with aortic dissection in Chinese men. Int J Cardiol 2016;202:196-7.

16. Li X, Jiang $\mathrm{S}, \mathrm{He} \mathrm{J}$, et al. Uric acid in aortic dissection: A meta-analysis. Clin Chim Acta 2018;484:253-7.

17. Hong Q, Qi K, Feng Z, et al. Hyperuricemia induces endothelial dysfunction via mitochondrial $\mathrm{Na}+/ \mathrm{Ca} 2+$ exchanger-mediated mitochondrial calcium overload. Cell Calcium 2012;51:402-10.

18. Esen AM, Akcakoyun M, Esen O, et al. Uric acid as a marker of oxidative stress in dilatation of the ascending aorta. Am J Hypertens 2011;24:149-54.

19. Huang B, Yang Y, Lu H, et al. Impact of d-Dimer Levels on Admission on Inhospital and Long-Term Outcome

Cite this article as: Yang G, Zhou Y, He H, Pan X, Li X, Chai $\mathrm{X}$. A nomogram for predicting in-hospital mortality in acute type A aortic dissection patients. J Thorac Dis 2020;12(3):264275. doi: $10.21037 /$ jtd.2020.01.41 in Patients With Type A Acute Aortic Dissection. Am J Cardiol 2015;115:1595-600.

20. Itagaki R, Kimura N, Mieno M, et al. Characteristics and Treatment Outcomes of Acute Type A Aortic Dissection With Elevated D-Dimer Concentration. J Am Heart Assoc 2018. doi: 10.1161/JAHA.118.009144.

21. Peng W, Zhu QY, Zhou XH, et al. A Simple Emergency Prediction Tool for Acute Aortic Dissection. Iran J Public Health 2013;42:1085-91.

22. Li Y, Zhong X, Cheng G, et al. Hs-CRP and all-cause, cardiovascular, and cancer mortality risk: A meta-analysis. Atherosclerosis 2017;259:75-82.

23. Vrsalović M, Vrsalovic PA. Admission C-reactive protein and outcomes in acute aortic dissection: a systematic review. Croat Med J 2019;60:309-15.

24. Duan XZ, Xu ZY, Lu FL, et al. Inflammation is related to preoperative hypoxemia in patients with acute Stanford type A aortic dissection. J Thorac Dis 2018;10:1628-34.

25. AlMahameed ST, Novaro GM, Asher CR, et al. Predictive value of high sensitivity $\mathrm{C}$-reactive protein in the diagnosis and outcomes of acute aortic syndromes. Heart Asia 2010;2:136-9.

26. Sakakura K, Kubo N, Ako J, et al. Peak C-reactive protein level predicts long-term outcomes in type B acute aortic dissection. Hypertension 2010;5 5:422-9.

27. Okina N, Ohuchida M, Takeuchi T, et al. Utility of measuring C-reactive protein for prediction of in-hospital events in patients with acute aortic dissection. Heart Vessels 2013;28:330-5. 